\title{
Ecología del paisaje y caracterización de la cobertura forestal de la microcuenca del Río La Balsa costa Rica
}

Marilyn Ortega-Rivera ${ }^{1}$ Braulio Vílchez-Alvarado²

\section{Resumen}

Se evaluó la ecología del paisaje, el cual está dominado por tres coberturas: bosques, pastos y cultivos. Las principales métricas de paisaje obtenidas: a) Bosque: Área de la clase (CA): 11447,72 ha; Número de Parches (NumP): 16344; Tamaño promedio de parches (MPS): 0,70 \pm 10,73 ha; Borde Total (TE): $5245378,35 \mathrm{~m}$; Borde promedio de parche (MPE): 320,94 m/ha; Índice de forma promedio (MSI): 1,47. b) Pastos: CA: 10467,63 ha; NumP: 13930; MPS: 0,75 $\pm 23,93$ ha; TE: 4432678,19 m; MPE: 318,21 m/ha; MSl: 1,47. c) Cultivos: CA: 3603,76 ha; NumP:636; MPS: 5,67 $\pm 63,59$ ha; TE: 7666342,78 m; MPE: 1204,94 m/ha; MSI: 1,93. Se obtuvo además un índice de diversidad de Shannon para el paisaje de 1,32. La composición florística y estructura se evaluaron en tres pisos altitudinales y para las principales coberturas forestales presentes. Se establecieron 68 parcelas temporales de $2000 \mathrm{~m}^{2}(20 \times 100 \mathrm{~m})$, identificándose las especies, la altura total y el diámetro de todos los árboles mayores a $10 \mathrm{~cm}$ de diámetro presentes en las parcelas de medición.

\begin{abstract}
Evaluated landscape ecology, which is dominated by three coverages: forests, pastures and crops. The main landscape metrics obtained: a) Forest: Class Area (CA): 11447,72 ha, Number of Patches (NumP): 16344, Mean Patch Size (MPS): 10,73 $\pm 0,70$ ha; Total Edge (TE): 5245378,35 m, Mean Patch Edge (MPE): 320,94 m / ha; Mean Shape Index (MSI): 1,47. b) Pasture: CA: 10467,63 ha; NumP: 13930; MPS: 23,93 $\pm 0,75$ ha, TE: 4,432,678,19 m; MPE: $318,21 \mathrm{~m} / \mathrm{ha}$, MSI: 1,47. c) Crops: CA: 3603,76 ha; NumP: 636; MPS: 63,59 $\pm 5,67$ ha, TE: 7,666,342,78 m; MPE: $1204,94 \mathrm{~m} / \mathrm{ha}, \mathrm{MSI}: 1,93$. Also obtained a diversity index for the landscape of 1,32 . The floristic composition and structure were evaluated in three altitudinal levels and major forest cover present. Sixty-eigth temporary plots were established of $2000 \mathrm{~m}^{2}(20 \times 100 \mathrm{~m})$, species were identified, total height was measured and diameter for all trees greater than $10 \mathrm{~cm}$. The mean values: a) Basal: disturbed forests, d: 23,3 cm, Ht: $24 \mathrm{~m}, \mathrm{~N}$ ha-1:
\end{abstract}


Los valores promedio obtenidos: a) Basal: bosques intervenidos, d: 23,3 cm; Ht: 24 m; N ha-1: 458 y G/ha: 22,8 $\mathrm{m}^{2}$. b) Premontano: bosques intervenidos, d: 22,6 cm; Ht: 27,3 m; N ha-1: 423 y G/ha: 20,6 m², potreros arbolados, d: 24,3 cm; Ht: 18,9 m; N ha-1: 121 y G/ha: $6,6 \mathrm{~m}^{2}$. c) Montano bajo: bosques de altura, d: 26,6 cm; Ht: 27,5 m; N ha-1: 380 y G/ha: 27,2 m², bosques intervenidos, d: 22,9 cm; Ht: $27 \mathrm{~m}$; N ha-1: 318 y G/ha: 15,8 $\mathrm{m}^{2}$, potreros arbolados, d: 24,5 cm; Ht: $25 \mathrm{~m}$; N ha-1: 118 y G/ha: $6,9 \mathrm{~m}^{2}$. La composición florística y las variables estructurales sugieren que los hábitats presentes en la microcuenca se encuentran en estado de recuperación.

Palabras clave: ecología del paisaje, cuencas, composición florística, estructura de bosque, pisos altitudinales, diversidad, Costa Rica.

\section{Introducción}

Los ecosistemas boscosos de la región Tropical, corresponden a los complejos biológicos más diversos de la biosfera, cuyos servicios suplen las necesidades de la sociedad y de los grupos humanos que allí habitan (Melo \& Vargas 2003), además proveen servicios a otros ecosistemas como: protección de los cauces, protección y retención de los suelos, producción de agua y mantenimiento de su calidad, protección de la biodiversidad, refugio y alimentación a la fauna, regulación de las zonas de recarga acuífera, entre otros.

En Centroamérica, la expansión de la frontera agrícola, la apertura de carreteras y la extracción de madera, está provocando la fragmentación acelerada de extensas áreas antes cubiertas por bosques naturales (Sánchez 2001). Diversas prácticas sociales y económicas generan una variedad de elementos dentro del paisaje, entre ellos los agropaisajes, que incluyen cortinas rompevientos, cercas vivas y linderos de vegetación, los cuales fueron establecidos por los productores para la protección contra el viento, el control de erosión, la provisión de madera o leña u otros propósitos (Harvey et al. 2004, 2005). La FAO (2003) ha estimado que el $40 \%$ de la totalidad del territorio Centroamericano está constituido por tierras utilizadas para pastoreo, a su vez, un $60 \%$ de estas áreas de pasturas se encuentran degradadas, como consecuencia de la producción ganadera tradicional basada en el manejo de pasturas sin árboles.

La transformación y fragmentación de los bosques tropicales representan la principal causa de la pérdida de diversidad biológica, se estima que cerca del $50 \%$ de los bosques tropicales han desaparecido en los últimos cincuenta años por acción de diferentes actividades de origen antrópico (Skoles et al. 1993, Jang et al. 1996; Gallego 2002). Específicamente, en Costa Rica entre
458 and G / ha: $22,8 \mathrm{~m}^{2}$. b) Premontane: disturbed forest, d: 22,6 cm, Ht: 27,3 m, N ha-1: 423 and G / ha: 20,6 m², wooded pastures, $\mathrm{d}: 24,3 \mathrm{~cm}, \mathrm{Ht}: 18,9 \mathrm{~m}, \mathrm{~N}$ ha-1: 121 and $\mathrm{G} /$ has: $6,6 \mathrm{~m}^{2} \mathrm{c}$ ) Lower montane: upland forests, d: 26,6 cm, Ht: 27,5 m, N ha-1: 380 and G / ha: 27,2 $\mathrm{m}^{2}$, disturbed forests, $\mathrm{d}: 22,9 \mathrm{~cm}$; $\mathrm{Ht}: 27 \mathrm{~m}, \mathrm{~N}$ ha-1: 318 and G /ha: $15,8 \mathrm{~m}^{2}$, wooded pastures, $\mathrm{d}: 24,5 \mathrm{~cm} \mathrm{Ht}: 25 \mathrm{~m}, \mathrm{~N}$ ha-1: 118 and $\mathrm{G} /$ has: $6,9 \mathrm{~m}^{2}$. The floristic composition and structural variables suggest the habitats present in the watershed are in recovery.

Key words: landscape ecology, watershed, floristic composition, forest structure, altitudinal, diversity, Costa Rica.

1986 y 1991 se perdieron 225000 ha de bosque húmedo tropical y húmedo premontano, alcanzando un promedio

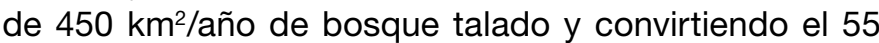
$\%$ de los bosques en fragmentos de 3 a 50 ha (Sánchez 2001). Esto ha ocasionado diferentes problemas ambientales, por un lado la deforestación provoca erosión y pérdida de la fertilización natural de los suelos, desertificación, emisión de gases de efecto invernadero y contaminación de aguas y por otro lado la fragmentación influye en el cambio de la composición de las especies de las comunidades bióticas y la alteración en la función de los ecosistemas (Kaimowitz 2001, Harvey et al. 2008). Asimismo, la pérdida contínua y el manejo inadecuado de estos hábitats afecta la calidad y disponibilidad de los servicios que dichos ecosistemas proveen, entre ellos, la producción y regulación del agua, fundamental en el desarrollo de gran cantidad de actividades productivas, en la que se destaca la producción de energía.

La Compañía Nacional de Fuerza y Luz, S.A. (CNFL) es una empresa dedicada a la venta y distribución de servicios eléctricos en la Meseta Central de Costa Rica; sin embargo, posee recursos hidroeléctricos fuera de ésta, asimismo es interés de dicha empresa desarrollar actividades de conservación y manejo integrado de cuencas, estrechamente asociado con el aprovechamiento y mejoramiento del recurso hídrico que llega a los embalses para la generación de energía hidroeléctrica. Como parte de sus proyectos de desarrollo, mejoramiento y distribución energética está el Proyecto Hidroeléctrico Balsa Inferior en la microcuenca del río La Balsa, cuenta con un área de 28638 ha y tendrá una potencia de $37,5 \mathrm{MW}$ (con tres unidades de $12,5 \mathrm{MW}$ ) y una producción promedio al año de $122 \mathrm{Gwh}$. Se requiere conocer y evaluar el estado y características de los ecosistemas que la componen y que permitan identificar los servicios ambientales que estos proporcionan; por lo que, el objetivo de este 
estudio es evaluar la ecología del paisaje y caracterizar la cobertura forestal de la microcuenca del Río La Balsa, Costa Rica, para el posterior establecimiento de criterios para el manejo integrado de la cuenca.

\section{Metodología}

Área de estudio: La microcuenca del Río La Balsa mide $286,4 \mathrm{~km}^{2}$, se localiza entre los cantones de San Ramón, San Carlos, Alfaro Ruíz y Naranjo, provincia de Alajuela entre las coordenadas $435000-460000 \mathrm{~m}$ este y 1122000 - 1147500 m norte (CRTM05). La precipitación promedio anual es de $1914 \mathrm{~mm}$, (Castro 2008), geomorfológicamente se caracteriza por presentar rangos altitudinales entre los $2300 \mathrm{~m}$ y los $100 \mathrm{~m}$ (Ramírez 2009), la pendiente media del cauce principal es de 15,7 $(\mathrm{m} / \mathrm{km})$ y la longitud es de $40 \mathrm{~km}$ (Castro 2008). Los usos del suelo predominantes son coberturas boscosas, usos de cultivos y pastos de diferentes tipos (Ramírez 2009) pertenecientes 3 a pisos altitudinales, basal, premontano y montano bajo, en los que se encuentran 10 zonas de vida, según el Sistema de clasificación de zonas de vida de Holdridge (1987).

Muestreo: Se utilizaron las capas de zona de vida según Holdridge (1987), ríos (CNFL), carreteras (ITCR 2008) y el mapa de cobertura del año 2008 basado en la interpretación de fotografías aéreas por parte del personal de la Compañía Nacional de Fuerza y Luz (CNFL).

A la capa de ríos y carreteras se les aplicó un buffer de 500 metros, se sobrepuso con el mapa de zonas de vida para obtener un mapa base, con el comando "point randomizer" del programa ArGis versión 9.3, se aleatorizaron los puntos de muestreo bajo los criterios de: no más de 12 puntos por zona de vida y con una intensidad del $1 \%$ del área total del mapa base, el cual contenía los usos de suelo: pastos arbolados y bosques. En cada punto de muestreo se estableció una parcela rectangular de 2000 $\mathrm{m}^{2}(20 \times 100 \mathrm{~m})$, para un total de 68 parcelas.

Recolección de datos: El muestreo inició a partir del mes de febrero del 2011. En cada parcela y para árboles con diámetro mayor o igual a $10 \mathrm{~cm}$ se midió: el diámetro a 1,3 m de altura, se estimó la altura total en metros $(\mathrm{m})$. La identificación taxonómica de individuos fue a nivel de familia, género y especie.

Análisis de datos: Para el análisis de la ecología del paisaje, se utilizaron los datos reportados en el mapa de cobertura del año 2008, respaldado por la respectiva comprobación de campo. Por otro lado, para el análisis de la composición florística y estructura, los datos se manejaron a nivel de piso altitudinal y sólo se tomaron en cuenta las principales coberturas forestales, en el caso de este estudio, potreros arbolados, bosques intervenidos y bosques de altura. Se utilizó la prueba de
Shapiro-Wilks para determinar la normalidad de los datos utilizando el programa estadístico InfoStat-e (Di Rienzo et al. 2011); sin embargo, al no encontrar normalidad se aplicó el método de interpercentiles (del $2 \%$ para cada cola) para homogenizar los datos a analizar.

\section{Variables}

Ecología del paisaje: Se utilizó la herramienta Patch Analyst Versión 2.1 (Elkie et al. 1999) que funciona como una extensión del software Arc View 3.3 (ESRI) que permite obtener diferentes métricas de fragmentación de paisaje. En este caso se evaluaron estadísticas a escala 1:150000, tanto a nivel de clase (fragmentos que representan el mismo uso de suelo), como a nivel de paisaje (para todos los fragmentos y clases a la vez), para las categorías de uso de suelo presentes en el paisaje, obtenidas a partir del mapa corregido de uso de suelo. Las métricas de paisaje analizadas por el software fueron: área total del paisaje (ha), área de la clase (ha), número de parches, tamaño promedio de parches (ha), desviación estándar de tamaño de parches (ha), borde total $(\mathrm{m})$, borde en relación con el área de paisaje $(\mathrm{m} /$ ha), borde promedio de parche ( $\mathrm{m} / \mathrm{ha})$, índice de forma promedio, índice de forma promedio pesado/área y el índice de diversidad de Shannon (a nivel de paisaje), dichas métricas se obtuvieron con la capa de usos de suelo en formato vector.

Composición florística: Se determinó el porcentaje del Índice de Valor de Importancia ( \% IVI) de Curtis y McIntosh (1951) citado por Lamprecht (1990), para cada especie por piso altitudinal, basado en los parámetros relativos de abundancia, frecuencia y dominancia. Se evaluaron patrones de diversidad por medio de índices basados en riqueza de especies y distribución espacial de los individuos (Shannon-Wiener, Inverso de Simpson y Alpha-Fisher), para el cálculo de los índices de diversidad se utilizó el programa PAST versión 2.09 (Hammer et al. 2001). Se efectuó un análisis de conglomerados por medio del software InfoStat-e (Di Rienzo et al. 2011), para resumir el comportamiento de las variables de estructura y composición florística para las diferentes coberturas forestales según el piso altitudinal. Para el análisis de diversidad, sólo se tomaron en cuenta las especies que se lograron identificar, por lo que no se tomaron en cuenta las especies desconocidas.

Estructura: la estructura fue analizada en su componente vertical y horizontal. Para la estructura horizontal, se evaluaron los valores promedio de diámetro, número de árboles $(\mathrm{N})$ y área basal $(\mathrm{G})$ en cada parcela según las diferentes coberturas forestales y para cada piso altitudinal. Para la estructura vertical de las unidades de muestreo se utilizó la metodología de IUFRO (Leibundgut 1958, citada por Lamprecht 1990), que define las alturas del piso inferior, medio y superior. 


\section{Resultados}

Ecología del paisaje: Los cuadros 1 y 2 muestran las métricas de paisaje analizadas para los principales usos de suelo encontrados en la microcuenca.

La mayor área la ocupan los bosques con 11447,7 ha, seguida por los pastos que abarcan 10467,6 ha. El uso urbano es, en conjunto con las plantaciones, las categorías que presentaron las áreas más bajas con 162 ha y 152,9 ha. En consecuencia, los bosques y los pastos presentan el mayor número de parches con 16344 y 13930, mientras que los cultivos ornamentales y el uso urbano presentan el menor número con 169 y 131 respectivamente.

Conforme aumenta el número de parches, disminuye el tamaño promedio de los mismos, se refleja en los valores presentados por los bosques $(0,7 \pm 10,7 \mathrm{ha}) \mathrm{y}$ pastos $(0,8 \pm 23,9$ ha). Las categorías urbano, cultivos y ornamentales presentan los tamaños de parche promedio más altos $(1,2 \pm 1,8$ ha, $5,7 \pm 63,6$ ha y $4,3 \pm 11,6$ ha respectivamente). Los tacotales, potreros arbolados y plantaciones presentan los valores más bajos $(0,2 \pm 0,6$ ha, $0,3 \pm 1,4$ ha y $0,5 \pm 0,4$ ha respectivamente) y se encuentran distribuidos en toda la matriz, que genera una importante red de recursos, que permite la distribución de muchas especies de plantas y animales.

Con respecto al borde total, el valor más alto está asociado al bosque $(5245378,4 \mathrm{~m})$, seguido por los pastos $(4432678,2 \mathrm{~m})$ y el valor mínimo lo presenta el uso urbano (89993,7 m). El borde promedio refleja cual es el perímetro correspondiente a cada uno de los parches por categoría de uso de suelo, entre más bajo sea el valor más "compacta" la forma, en este caso los tacotales (220,1 mha-1) y los pastos arbolados $(234,17$ mha- 1 ) y las categorías que poseen formas menos compactas son los cultivos, con un promedio de 1204,94 mha-1 y los
Cuadro 1. Métricas de fragmentación para las categorías de uso de suelo presentes en el paisaje de la microcuenca del Río La Balsa, Costa Rica.

\begin{tabular}{|l|l|l|l|l|}
\hline \multicolumn{1}{|c|}{ Métricas* } & \multicolumn{1}{|c|}{ Pastos } & \multicolumn{1}{c|}{ Urbano } & \multicolumn{1}{|c|}{ Cultivos } & Ornamentales \\
\hline CA (ha) & 10467,63 & 162,01 & 3603,76 & 730,64 \\
\hline NumP & 13930,00 & 131,00 & 636,00 & 169,00 \\
\hline MPS (ha) & 0,75 & 1,24 & 5,67 & 4,32 \\
\hline PSSD (ha) & 23,93 & 1,79 & 63,59 & 11,61 \\
\hline TE (m) & 4432678,19 & 89993,66 & 766342,78 & 175437,46 \\
\hline ED (m/ha) & 197,20 & 3,24 & 27,59 & 6,32 \\
\hline MPE (m/ha) & 318,21 & 686,97 & 1204,94 & 1038,09 \\
\hline MSI & 1,47 & 1,76 & 1,93 & 1,71 \\
\hline AWMSI & 10,87 & 2,30 & 7,11 & 2,80 \\
\hline
\end{tabular}

CA: Área de la clase, NumP: Número de parches, MPS: Tamaño promedio de parches, PSSD: Desviación estándar de tamaño de parches, TE: Borde total, ED: Borde en relación con el área de paisaje, MPE: Borde promedio de parche, MSI: Índice de forma promedio, AWMSI: Índice de forma promedio pesado/área.

cultivos de ornamentales con 1038,09 mha-1.

El valor del MSI es 1, entre más lejano a 1 es el valor, más compleja es la forma del parche, en este caso a nivel de categoría todas presentan valores cercanos a 1 , en un intervalo que va de 1,4 para el tacotal a 1,9 para los cultivos.

Si se analiza este índice a nivel de área total por categoría, se tiene que, las categorías de uso con mayor área (pastos, cultivos y bosques), presentaron los valores de Índice de Forma más altos con 10,9, 7,1 y 5,9 respectivamente, lo que permite inferir que a mayor área, más compleja es la forma de los parches, Sin embargo, se deben tomar en cuenta otras coberturas que presentan formas menos complejas, pero que generan un recurso adicional a la conectividad, como lo son los tacotales, potreros arbolados y plantaciones con valores de 1,8, 2,9 y 2 respectivamente.

Cuadro 2. Métricas de fragmentación a nivel de paisaje y para las coberturas forestales presentes en el paisaje de la microcuenca del Río La Balsa, Costa Rica.

\begin{tabular}{|c|c|c|c|c|c|}
\hline Métricas* & Tacotal & Bosque & Pastos arbolados & Plantación & A nivel de paisaje \\
\hline TLA (ha) & & & & & 27774,72 \\
\hline CA (ha) & 800,72 & 11447,72 & 409,36 & 152,89 & 27774,72 \\
\hline NumP & 3329,00 & 16344,00 & 1486,00 & 283,00 & 36308,00 \\
\hline MPS (ha) & 0,24 & 0,70 & 0,28 & 0,54 & 0,76 \\
\hline PSSD (ha) & 0,62 & 10,73 & 1,42 & 0,42 & 18,54 \\
\hline $\mathrm{TE}(\mathrm{m})$ & 732558,35 & 5245378,35 & 347977,30 & 130276,99 & 11920643,07 \\
\hline ED (m/ha) & 26,38 & 233,36 & 15,48 & 5,80 & 429,19 \\
\hline MPE (m/ha) & 220,05 & 320,94 & 234,17 & 460,34 & 328,32 \\
\hline $\mathrm{MSI}$ & 1,41 & 1,47 & 1,50 & 1,78 & 1,48 \\
\hline AWMSI & 1,87 & 5,88 & 2,97 & 2,00 & 7,64 \\
\hline$H^{\prime}$ & & & & & 1,32 \\
\hline
\end{tabular}

*TLA: Área total del paisaje, CA: Área de la clase, NumP: Número de parches, MPS: Tamaño promedio de parches, PSSD: Desviación estándar de tamaño de parches, TE: Borde total, ED: Borde en relación con el área de paisaje, MPE: Borde promedio de parche, MSI: Índice de forma promedio, AWMSI: Índice de forma promedio pesado/área, H': Índice de Shannon. 
Cuadro 3. Porcentaje del Índice de Valor de Importancia (\% IVI), para las 10 especies de mayor peso ecológico en el piso altitudinal basal en la microcuenca del Río La Balsa, Costa Rica.

\begin{tabular}{|c|c|c|c|c|}
\hline \multicolumn{1}{|c|}{ Especie } & \multirow{2}{*}{ *AR (\%) } & DR (\%) & FR (\%) & $\begin{array}{c}\text { I.V.I ( } \\
\%)\end{array}$ \\
\hline Cordia alliodora & 6,00 & 6,65 & 2,25 & 14,90 \\
\hline Prunus annularis & 6,55 & 4,78 & 1,12 & 12,45 \\
\hline Chimarrhis parviflora & 3,64 & 4,15 & 1,12 & 8,91 \\
\hline Erythrina costaricensis & 2,36 & 5,13 & 1,12 & 8,61 \\
\hline Iriartea deltoidea & 4,73 & 2,71 & 1,12 & 8,56 \\
\hline Inga edulis & 2,73 & 2,43 & 2,25 & 7,41 \\
\hline Cecropia peltata & 2,18 & 3,08 & 1,69 & 6,95 \\
\hline Vochysia guatemalensis & 1,82 & 3,86 & 1,12 & 6,81 \\
\hline Apeiba membranacea & 2,00 & 1,86 & 2,81 & 6,67 \\
\hline Sapium glandulosum & 1,64 & 2,59 & 2,25 & 6,47 \\
\hline
\end{tabular}

*AR: Abundancia Relativa, DR: Dominancia Relativa, FR: Frecuencia Relativa.

Finalmente, se obtuvo un Índice de diversidad de Shannon $\left(H^{\prime}\right)$ a nivel de paisaje de 1,3; valor medianamente bajo, donde el mosaico paisajístico está dominado por 3 coberturas, cultivos, pastos y bosques, en el que circunscriben otras categorías que responden a la distribución de los recursos en el paisaje.

Composición florística: se identificaron un total de 406 especies de árboles, correspondientes a 3 pisos altitudinales, representando a 79 familias y 208 géneros.

Índice de Valor de Importancia (IVI): El cuadro 3 muestra los valores obtenidos para el piso basal (Cuadro 3).

Las especies Cordia alliodora (Ruiz \& Pav.) Oken y Cordia alliodora Koehne, fueron las especies de mayor peso ecológico, con porcentajes relativamente bajos (14,9\% y $12,5 \%$ respectivamente. En el piso premontano, el cuadro 4 muestra que la especie $C$. alliodora presentó el mayor porcentaje para el IVI, seguida de las especies Cedrela odorata L. y Miconia argentea (Sw.) DC., todas representativas de bosques intervenidos o potreros arbolados de la parte media y alta de la microcuenca.

El cuadro 5 resume los valores para el piso montano bajo, las especies con mayor peso ecológico corresponden a los bosques que dominaron los valores de área basal, las especies Quercus seemannii Lieb., Drymis granadensis L. f. y Quercus corrugata Hooker, especies características de los bosques de altura representativos de este piso altitudinal.

Con los valores de IVI obtenidos en cada piso altitudinal, se denota que especies como $C$. alliodora, $P$. annularis y $M$. argentea presentan una amplia distribución altitudinal y que el piso montano bajo está dominado principalmente por especies del género Quercus spp.
Cuadro 4. Porcentaje del Indice de Valor de Importancia (\% IVI), para las 10 especies de mayor peso ecológico en el piso altitudinal premontano en la microcuenca del Río La Balsa, Costa Rica.

\begin{tabular}{|c|c|c|c|c|}
\hline Especie & ${ }^{*}$ AR (\%) & DR (\%) & FR (\%) & I.V.I (\%) \\
\hline Cordia alliodora & 9,598 & 8,247 & 2,388 & 20,234 \\
\hline Cedrela odorata & 2,547 & 4,376 & 1,343 & 8,265 \\
\hline Miconia argentea & 2,253 & 2,285 & 1,940 & 6,478 \\
\hline Prunus annularis & 3,477 & 2,642 & 0,299 & 6,418 \\
\hline Hampea appendiculata & 1,567 & 3,537 & 1,194 & 6,298 \\
\hline Heliocarpus appendiculatus & 2,449 & 1,935 & 1,194 & 5,578 \\
\hline Cecropia insignis & 1,861 & 1,399 & 2,090 & 5,350 \\
\hline Dendropanax arboreus & 1,959 & 1,552 & 1,791 & 5,302 \\
\hline Ficus goldmanii & 1,616 & 2,792 & 0,746 & 5,154 \\
\hline Cecropia peltata & 1,812 & 1,675 & 0,896 & 4,382 \\
\hline
\end{tabular}

*AR: Abundancia Relativa, DR: Dominancia Relativa, FR: Frecuencia Relativa.

Índices de riqueza y diversidad: El índice de ShannonWiener $\left(\mathrm{H}^{\prime}\right)$ promedio para los bosques intervenidos en el piso basal fue de 3,00, presentándose como el hábitat más diverso. Para el piso premontano, se reportan valores de 2,96 y 1,37 para los bosques intervenidos y potreros arbolados respectivamente. En el montano bajo, los valores más altos los presentan los bosques intervenidos con un valor de 2,56; seguido por los potreros arbolados con 2,44 y los bosques de altura con 2,33.

Para el inverso de Simpson, los bosques intervenidos del piso basal se presentan como los más diversos, con un valor de 0,93. Los bosques intervenidos y potreros arbolados del piso premontano presentaron valores de 0,92 y 0,63 respectivamente. Para el piso montano bajo, los potreros arbolados presentaron la mayor diversidad, con un valor de 0,89 , seguidos por los bosques intervenidos $(0,88)$ y los bosques de altura $(0,87)$.

Cuadro 5. Porcentaje del Índice de Valor de Importancia (\% IVI), para las 10 especies de mayor peso ecológico en el piso altitudinal montano bajo en la microcuenca del Río La Balsa, Costa Rica.

\begin{tabular}{|c|c|c|c|c|}
\hline Especie & *AR ( \%) & DR ( \%) & FR (\%) & $\begin{array}{c}\text { I.V.I ( } \\
\%)\end{array}$ \\
\hline Quercus seemannii & 7,83 & 17,76 & 3,45 & 29,03 \\
\hline Drimys granadensis & 12,95 & 6,91 & 3,74 & 23,59 \\
\hline Quercus corrugata & 4,65 & 11,84 & 3,45 & 19,94 \\
\hline Miconia argentea & 4,81 & 2,37 & 4,31 & 11,49 \\
\hline Cornus disciflora & 4,03 & 4,34 & 2,59 & 10,96 \\
\hline Dendropanax arboreus & 3,49 & 3,25 & 3,45 & 10,19 \\
\hline Roupala montana & 4,19 & 2,76 & 3,16 & 10,11 \\
\hline Oreopanax xalapensis & 2,79 & 2,81 & 3,45 & 9,05 \\
\hline Trichilia americana & 3,26 & 1,84 & 3,74 & 8,83 \\
\hline Nectandra salicina & 2,79 & 3,02 & 2,59 & 8,40 \\
\hline
\end{tabular}

*AR: Abundancia Relativa, DR: Dominancia Relativa, FR: Frecuencia Relativa 
El cuadro 6 resume los valores de índices de diversidad de Shannon-Wiener $\left(H^{\prime}\right)$, inverso de Simpson (1-D) y Alpha Fisher, para las diferentes coberturas.

En cuanto al índice Alpha de Fisher a diferencia de los otros índices evaluados, el valor de diversidad no se ve afectado por la abundancia de especies raras (con un solo individuo) o especies muy abundantes (Medianero et al. 2003). En el piso basal, se reportó un valor de 18,01 para los bosques intervenidos. Los bosques intervenidos del piso premontano presentaron el valor más alto con base en este índice con 19,04; mientras que los poteros arbolados asociados a este piso reportaron un valor de 4,67. El ecosistema más diverso, en el piso montano bajo está representado por los potreros arbolados con un valor de 13,25; mientras que los bosques intervenidos y los bosques de altura presentaron valores de 9,63 y 6,04 respectivamente.

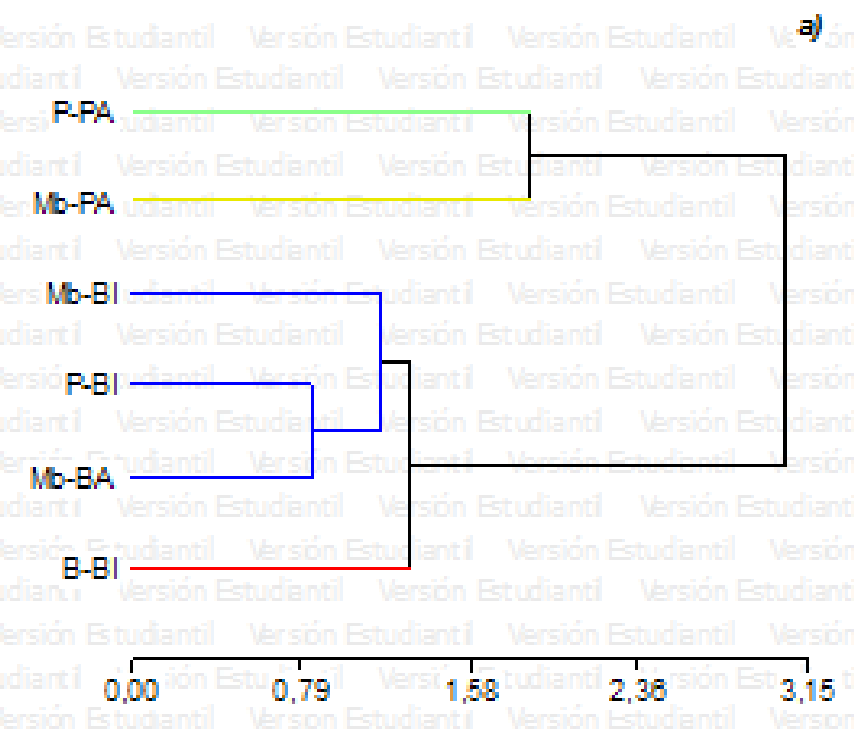

Análisis de conglomerados: La figura 1 muestra el análisis de conglomerados para las variables de estructura (a) y composición (b) para las diferentes coberturas forestales y según piso altitudinal.

Considerando sólo las variables de estructura $(\mathrm{N}, \mathrm{G}$ y $\mathrm{Ht}$ ), el conglomerado hace dos grupos principales. Los potreros arbolados evaluados en el premontano y montano bajo conformaron un conglomerado, que coincidió con la cobertura con el menor desarrollo estructural. El segundo grupo se compuso de los bosques evaluados en los diferentes pisos altitudinales, siento esta cobertura la que presenta individuos con mayores dimensiones, mayor densidad y número de árboles.

Para el análisis de la composición florística, se aplicó el mismo procedimiento, las variables analizadas fueron los índices de diversidad, en este caso, se identificaron

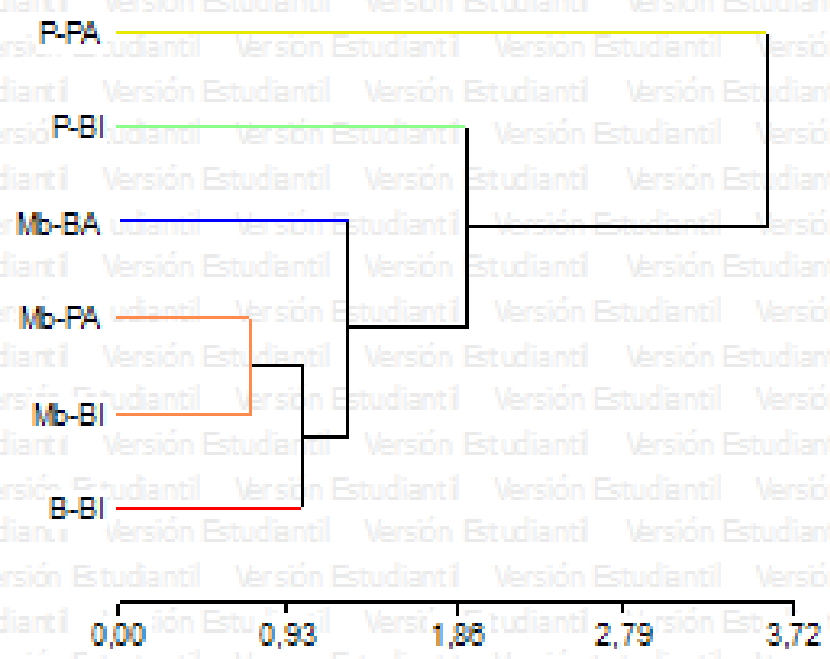

** B: basal, P: premontano, Mb: montano bajo; *BI: bosque intervenido, BA: bosque de altura, PA: potrero arbolado.

Fig 1. Análisis de conglomerados para las variables de estructura (a) y composición florística (b), para las diferentes coberturas forestales (bosque intervenido, bosque de altura, potrero arbolado) según piso altitudinal (basal, premontano y montano bajo) en la microcuenca del río La Balsa, Costa Rica.

Cuadro 6. Valores promedio (Media) y desviación estándar (DesvEst) de los índices de diversidad de Shannon-Wiener (H'), inverso de Simpson (1-D) y Alpha Fisher, para las diferentes coberturas forestales* según piso altitudinal en la microcuenca del río La Balsa, Costa Rica.

\begin{tabular}{|c|c|c|c|c|c|c|}
\hline \multirow{2}{*}{ Piso altitudinal } & \multicolumn{2}{|c|}{ Shannon-Wiener $(\mathrm{H})$} & \multicolumn{2}{|c|}{ Inverso de Simpson (1-D) } & \multicolumn{2}{|c|}{ Alpha-Fisher } \\
\hline & Media & DesvEst & Media & DesvEst & Media & DesvEst \\
\hline \multicolumn{7}{|l|}{ Basal } \\
\hline $\mathrm{Bl}$ & 3,00 & 0,40 & 0,93 & 0,03 & 18,01 & 10,98 \\
\hline \multicolumn{7}{|l|}{ Premontano } \\
\hline $\mathrm{Bl}$ & 2,96 & 0,37 & 0,92 & 0,04 & 19,04 & 12,01 \\
\hline $\mathrm{PA}$ & 1,37 & 0,74 & 0,63 & 0,26 & 4,67 & 5,13 \\
\hline \multicolumn{7}{|l|}{ Montano bajo } \\
\hline $\mathrm{BA}$ & 2,33 & 0,37 & 0,87 & 0,05 & 6,04 & 2,32 \\
\hline $\mathrm{Bl}$ & 2,56 & 0,34 & 0,88 & 0,08 & 9,63 & 3,18 \\
\hline $\mathrm{PA}$ & 2,44 & 0,06 & 0,89 & 0,02 & 13,25 & 0,15 \\
\hline
\end{tabular}

*BI: Bosque intervenido, BA: Bosque de altura, PA: Potrero arbolado. 
tres grupos: el primero correspondió a las coberturas evaluadas en el piso premontano. El segundo las coberturas evaluadas en el piso montano bajo. El tercero los bosques evaluados en el piso basal. El comportamiento que propone el conglomerado evidencia la transición de las especies según el gradiente altitudinal.

\section{Estructura horizontal}

Diámetro: Ios bosques intervenidos evaluados en el piso altitudinal basal, presentaron un diámetro promedio de 23,3 \pm 9,5 cm. Para el piso premontano los bosques intervenidos reportaron diámetros promedio 22,6 6 10,5 cm y los potreros arbolados valores de 24,7 $\pm 10,2 \mathrm{~cm}$. En el piso montano bajo se presentó un diámetro promedio de 26,6 \pm 14,2 cm para los bosques de altura, 22,9 \pm 10,2 cm para los bosques intervenidos y 24,5 $\pm 12,8 \mathrm{~m}$ para los potreros arbolados. Los valores de diámetro, altura, número de árboles y área basal para las diferentes coberturas forestales se resumen en el cuadro 7.

Número de individuos: los bosques intervenidos en el piso basal presentaron los valores promedio más altos para esta variable, con $458 \pm 249$ árboles/ha.

Los bosques intervenidos y potreros arbolados del piso premontano mostraron valores de $423 \pm 134$ árboles/ ha y $121 \pm 51$ árboles/ha respectivamente. Para el piso montano bajo se presentaron los valores más bajos, con $380 \pm 128$ árboles/ha para los bosques de altura, $318 \pm 104$ árboles/ha para los bosques intervenidos y $118 \pm 11$ árboles/ha para los potreros arbolados.

Distribución diamétrica ( $\mathbf{N} \mathbf{h a}^{-1}$ ): Para todas las coberturas (bosque intervenido, bosque de altura y potreros arbolados), en los diferentes pisos altitudinales (basal, premontano y montano bajo) se sigue un comportamiento de "J" invertida, donde la acumulación de individuos se da en las clases diamétricas de 10$20 \mathrm{~cm}$ y $20-30 \mathrm{~cm}$, con pocos individuos mayores a
$60 \mathrm{~cm}$. La figura 2 reúne la distribución diamétrica del número de individuos promedio por hectárea, para las diferentes coberturas forestales según el piso altitudinal basal, premontano y montano bajo en la microcuenca del Río La Balsa, Costa Rica.

Área basal: en el piso basal, los bosques intervenidos reportaron un valor promedio de 22,8 $\pm 12,8 \mathrm{~m}^{2} / \mathrm{ha}$. Para el piso premontano, los bosques intervenidos y potreros arbolados presentan valores de $20,6 \pm 5,9 \mathrm{~m}^{2} / \mathrm{ha}$ y 6,6 $\pm 2,7 \mathrm{~m}^{2} /$ ha respectivamente. En el piso montano bajo, los bosques intervenidos presentaron un valor promedio de 15,7 $\pm 4,3 \mathrm{~m}^{2} /$ ha, los bosques de altura presentaron un área basal promedio de 27,2 $\pm 11,2 \mathrm{~m}^{2} / \mathrm{ha}$, la mayor presentada para esta variable y los potreros arbolados un promedio de $6,9 \pm 1,1 \mathrm{~m}^{2} /$ ha. La figura 3 contiene la distribución diamétrica del área basal promedio por hectárea, para las diferentes coberturas forestales según el piso altitudinal basal, premontano y montano bajo en la microcuenca del Río La Balsa, Costa Rica.

\section{Estructura vertical}

Altura total: los bosques intervenidos evaluados en el piso altitudinal basal, presentaron una altura total promedio de 24,0 \pm 4,2 $\mathrm{m}$. Para el piso premontano los bosques intervenidos reportaron una altura promedio de $27,3 \pm 5,5 \mathrm{~m}$ y los potreros arbolados una altura total promedio de 18,8 $\pm 5,0 \mathrm{~m}$. En el piso montano bajo se reportó una altura de $27,5 \pm 3,8 \mathrm{~m}$ para los bosques de altura, 27,0 $\pm 4,2 \mathrm{~m}$ para los bosques intervenidos y 25,0 $\pm 7,1 \mathrm{~m}$ para los potreros arbolados.

Estratos de altura: En función de las alturas máximas, se determinaron los estratos de altura. La figura 4 resume la distribución del número de individuos promedio por hectárea, por estrato de altura, para las diferentes coberturas forestales, según piso altitudinal basal, premontano y montano bajo en la microcuenca del Río La Balsa, Costa Rica.

Cuadro 7. Promedio y desviación estándar (DesvEst) de las variables diámetro (d), altura total (Ht), número de árboles (N) y área basal (G), para las diferentes coberturas forestales * según piso altitudinal en la microcuenca del río La Balsa, Costa Rica.

\begin{tabular}{|c|c|c|c|c|c|c|c|c|}
\hline \multirow{2}{*}{ Piso altitudinal } & \multicolumn{2}{|c|}{$d(\mathrm{~cm})$} & \multicolumn{2}{|c|}{$\mathrm{Ht}(\mathrm{m})$} & \multicolumn{2}{|c|}{$\mathrm{N}$ ha-1 } & \multicolumn{2}{|c|}{$\mathrm{G}\left(\mathrm{m}^{2} / \mathrm{ha}\right)$} \\
\hline & Media & DesvEst & Media & DesvEst & Media & DesvEst & Media & DesvEst \\
\hline \multicolumn{9}{|l|}{ Basal } \\
\hline $\mathrm{Bl}$ & 23,33 & 9,45 & 24,00 & 4,20 & 458 & 249 & 22,80 & 12,81 \\
\hline \multicolumn{9}{|l|}{ Premontano } \\
\hline $\mathrm{Bl}$ & 22,55 & 10,53 & 27,32 & 5,46 & 423 & 134 & 20,56 & 5,92 \\
\hline PA & 24,27 & 10,20 & 18,83 & 5,02 & 121 & 51 & 6,59 & 2,73 \\
\hline \multicolumn{9}{|l|}{ Montano bajo } \\
\hline BA & 26,63 & 14,20 & 27,50 & 3,780 & 380 & 128 & 27,18 & 11,18 \\
\hline $\mathrm{Bl}$ & 22,96 & 10,17 & 27,00 & 4,22 & 318 & 104 & 15,72 & 4,25 \\
\hline PA & 24,45 & 12,77 & 25,00 & 7,07 & 118 & 11 & 6,99 & 1,12 \\
\hline
\end{tabular}

*BI: Bosque intervenido, BA: Bosque de altura, PA: Potrero arbolado. 


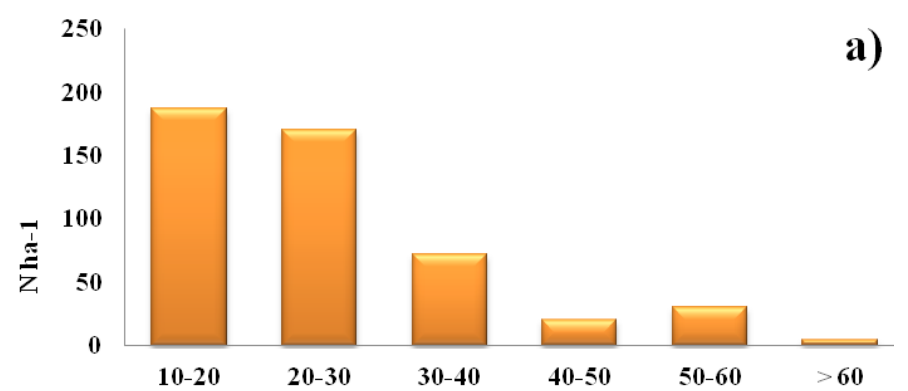

Categorias diamétricas (cm)
Gosqueintervenido


Fig 2. Distribución diamétrica del número de individuos promedio por hectárea ( $N$ ha-1), para las diferentes coberturas forestales según el piso altitudinal basal (a), premontano (b) y montano bajo (c) en la microcuenca del río La Balsa, Costa Rica

Para el piso basal, los estratos de altura de dosel son 8,16 y 24 $\mathrm{m}$ para los pisos inferior, medio y superior, respectivamente, en este caso los bosques intervenidos presentan más del 60 $\%$ de individuos en el estrato medio del dosel.

En el caso de los bosques intervenidos y potreros arbolados evaluados en el piso premontano, los estratos de altura son 31,21 y 10 respectivamente para ambas coberturas y al igual que en los otros pisos evaluados, la mayor cantidad de individuos se ubica en el estrato medio (56 \% y $44 \%$ respectivamente), seguido por aproximadamente un $30 \%$ en el estrato inferior y un 10 $\%$ y $24 \%$ en el estrato superior.



Categorías diamétricas (cm)


Fig 3. Distribución diamétrica del área basal promedio por hectárea (G) ha), para las diferentes coberturas forestales según el piso altitudinal basal (a), premontano (b) y montano bajo (c) en la microcuenca del río La Balsa, Costa Rica

En el piso montano bajo, tanto los bosques de altura, como los bosques intervenidos, con estratos de altura de dosel de 10,20 y $30 \mathrm{~m}$, acumulan la mayor cantidad de individuos en el estrato medio, seguido aproximadamente por un $30 \%$ en el dosel inferior y menos del $20 \%$ en el estrato superior; sin embargo, esta tendencia no se presenta para los potreros arbolados en este piso, que presenta estos de altura menores (7 y $21 \mathrm{~m})$.

En resumen, el dosel que alcanzó mayor altura fue el de los bosques intervenidos presentes en el piso premontano, sin embargo, los bosques intervenidos del piso basal presentaron la mayor cantidad de individuos en el dosel superior, aunque con una altura menor (24 m), 
mientras que los bosques y potreros arbolados del piso montano bajo mostraron los valores de altura promedio más altos.

\section{Discusión}

\section{Ecología del paisaje}

Si bien, el paisaje está dominado por pastos y dedicado a la producción ganadería extensiva y de doble propósito (producción de carne y leche), mantiene una cobertura arbórea heterogénea, compuesta de pequeños fragmentos de bosque, franjas angostas de bosques riparios, árboles dispersos en las pasturas y cercas vivas. Lo que se da en diferentes densidades y distribuciones espaciales.

En la microcuenca, los bosques cubren aproximadamente el $40 \%$ del paisaje, compuestos por parches que en promedio no superan 1 ha, pero poseen formas que facilitan la conectividad. Atributos como el tamaño, la forma, la distancia entre fragmentos, densidad de borde, calidad de los elementos, grado de naturalidad de los parches, son entre otros, factores importantes de una red de conexión física que facilita la dinámica de las metapoblaciones creando redes continuas de recursos que abastecen a gran variedad de ecosistemas.

Un ejemplo corresponde a los árboles dispersos y cercas vivas, coberturas predominantes dentro de la microcuenca, importantes para la conservación de especies de animales y plantas, ya que aumentan la cobertura arbórea total, dividen las pasturas en áreas más pequeñas, crean redes lineales que cruzan el paisaje y ofrecen conexiones físicas directas a los parches de bosque, con lo que facilitan el movimiento de algunas especies (Chacón \& Harvey 2008). Sin embargo, la reducción o desaparición de hábitats naturales supone no sólo un desbalance en la dinámica natural de las poblaciones, sino que tiene una influencia directa en la funcionalidad de la red hídrica, ya que, al disminuir dichas áreas, se supone un descenso en la recarga de los acuíferos, una disminución de la calidad del agua, una mayor frecuencia de las avenidas extremas (Morera et al. 2007) y afecta directamente las áreas de captación de agua, ya que al reducir la cobertura vegetal, la erosión aumenta, y el transporte de sedimentos en las partes bajas de las cuencas se hace más evidente (Saunders et al. 1991), además condicionan un aumento en la impermeabilización del suelo, Iroumé y Huber (2000) señalan que la producción hídrica puede ser aumentada al reducir la cubierta boscosa y arbustiva. Por lo tanto, la sustitución parcial de los bosques estudiados por una cubierta herbácea conduciría a un aumento de la cantidad de agua que percolaría en estos suelos. Dicho reemplazo derivaría de una reducción de las pérdidas por intercepción y por transpiración (Huber et al. 2007).

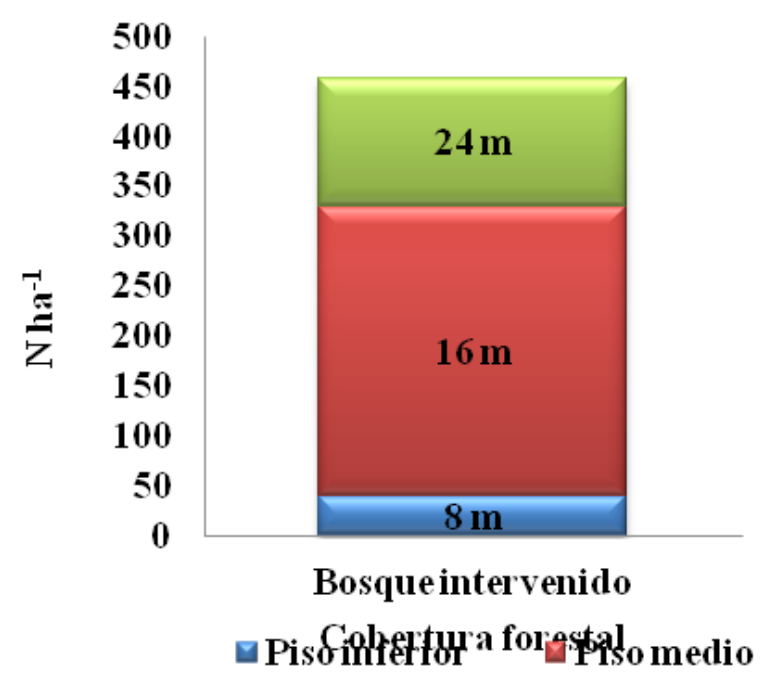

a)
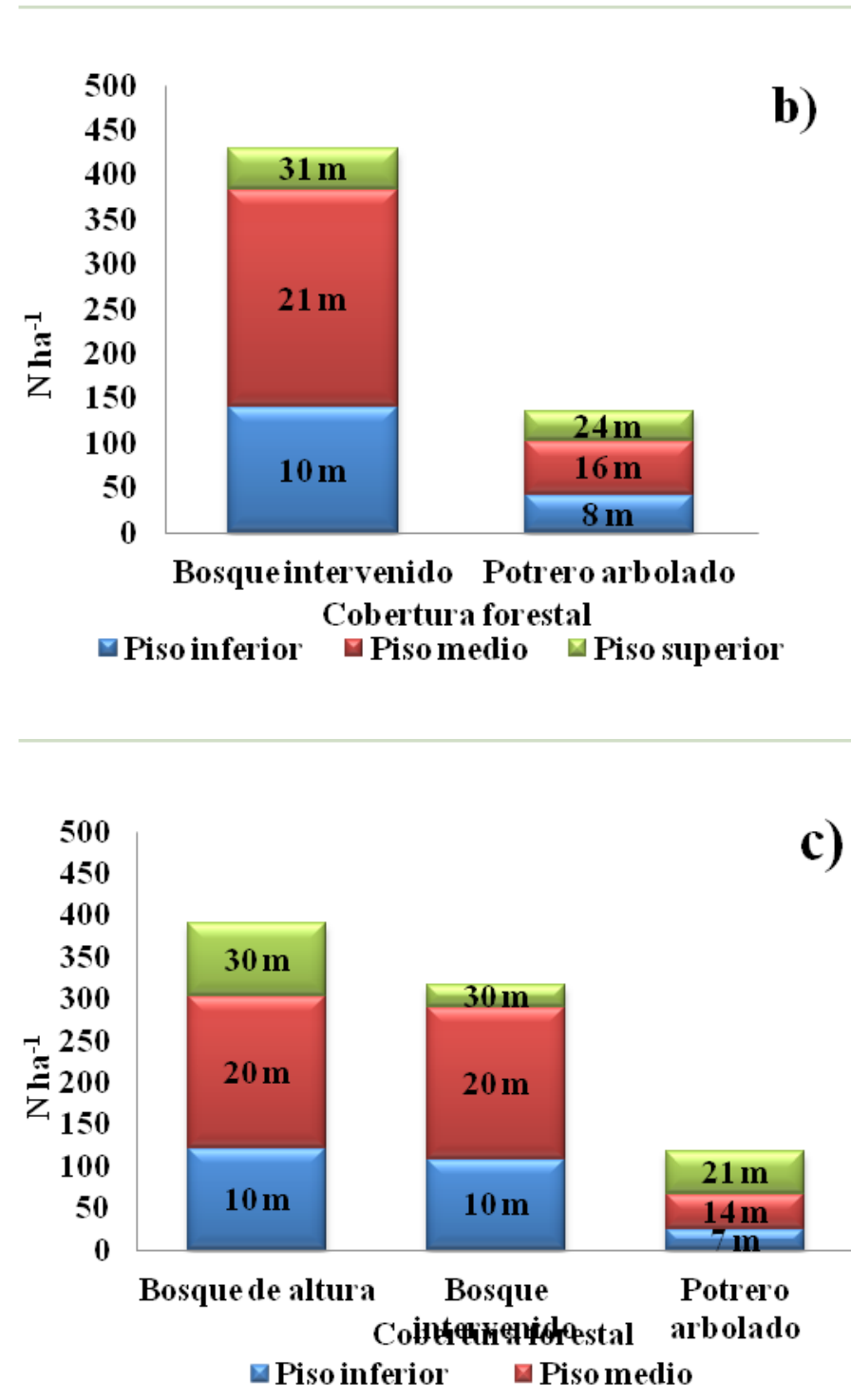

Fig 4. Distribución del número de individuos promedio por hectárea (N ha-1) por estrato de altura (piso inferior, piso medio y piso superior), para las diferentes coberturas forestales, según piso altitudinal basal (a), premontano (b) y montano bajo (c) en la microcuenca del Río La Balsa, Costa Rica. 
Es fundamental evaluar los elementos que componen la matriz del paisaje, en este caso particular, permiten crear un panorama más amplio acerca de la distribución de los recursos, las condiciones que prevalecen, las áreas que requieren un manejo prioritario y el manejo de los recursos a favor de la estabilidad ecológica, funcional y estructural de la microcuenca, con el fin de asegurar la disponibilidad y calidad del agua tanto para consumo humano, como para la generación hidroeléctrica.

\section{Composición florística}

En el piso altitudinal basal se encontraron especies representativas de bosques intervenidos entre ellas las más abundantes fueron C. alliodora, P. annularis, Chimarrhis parviflora Standley \& L.O. Williams, Cecropia peltata L., Apeiba membranacea Spruce ex Benth, Vochysia guatemalensis J.D.Smith, Iriartea deltoidea (Ruiz \& Pav.), composición similar a la encontrado por Quesada (2003) en bosques intervenidos de bajura.

El piso premotano presenta ecosistemas donde la especie que está más representada es, C. alliodora, seguida de la especie $C$. odorata. Se encuentran también otras especies típicas de crecimiento en claros, como Cecropia insignis Liebm. y Heliocarpus appendiculatus Turcz, mismas encontradas por Cascante y Estrada (2001) en claros de bosque húmedo premontano en el Valle Central.

Para el piso montano bajo, los bosques de altura y los intervenidos, están dominados por las especies $Q$. seemannii y $Q$. corrugata y gran cantidad de especies de la familia Lauraceae, al igual que los bosques estudiados por Nadkarni et al. (1995) en la Reserva de Bosque Nuboso Monteverde. Según Kappelle (2001), en estudios realizados en bosques montanos de Costa Rica, estas comunidades se caracterizan por tener hasta cinco estratos diferentes, con el dosel dominado por una o dos especies del género Quercus y frecuentemente asociado a especies como D. granadensis, Nectandra spp, Ocotea spp, Miconia spp, Roupala montana Aubl., Ilex pallida Standl., Guarea tonduzii C.DC., Trichilia havanensis Jacq. El subdosel incluye especies como Ardisia spp, Cornus disciflora DC., Dendropanax querceti Donn. Sm., Oreopanax spp y $P$. annularis. Los bosques estudiados comparten la presencia de muchas de estas especies y géneros, además de la abundante presencia de palmas, bambúes y helechos arborescentes (género Alsophila spp).

A nivel general, la estructura y composición florística de las coberturas forestales evaluadas en la microcuenca de Río La Balsa varía según las condiciones bióticas y abióticas predominantes; en el caso de la estructura se divide en dos grandes grupos, los bosques y los potreros arbolados, siendo éstos últimos los ecosistemas menos diversos, con menos de 20 especies; y donde el bosque intervenido representa la cobertura más diversa en todos los pisos altitudinales evaluados. Dicha diversidad sigue una tendencia marcada, aumenta conforme disminuye el gradiente altitudinal.

El análisis de la composición florística permite evaluar la influencia de la misma dentro de la dinámica hidrológica de la microcuenca, ya que la cantidad de precipitación que llega a la superficie, depende en gran medida del tipo y densidad de la cubierta vegetal lo que aumenta la importancia del estudio y manejo de la composición florística existente en la microcuenca.

\section{Estructura horizontal}

En diversos estudios realizados en bosques muy húmedos tropicales, se han encontrado densidades que van desde los 395 a 553 árboles/ha (Lieberman et al. 1990, Thomsen 1997, Acosta 2012, Morales 2010, Vílchez et al. 2008). Los bosques intervenidos correspondientes al piso altitudinal basal presentan un número de individuos que se asemeja al reportado en los estudios mencionados con 458 árboles/ha (diámetros mayores a $10 \mathrm{~cm}$ dap). A nivel de área basal las variaciones existentes, con respecto a los trabajos reportados y el valor observado para los bosques intervenidos en el piso altitudinal basal (23,3 $\mathrm{m}^{2} / \mathrm{ha}$ ) permite inferir que dichos ecosistemas han sido altamente intervenidos, sin embargo, la distribución de los individuos y de área basal según clases diamétricas garantiza una posible recuperación, ya que más del $70 \%$ de los individuos se encuentra en las clases que van de 10 a $30 \mathrm{~cm}$, lo que asegura una reserva de árboles que reemplazará los individuos que mueren, en ese sentido, el "rendimiento sostenido natural", está obviamente asegurado (Lamprecht 1990).

En cuanto a los bosques intervenidos del piso premontano, el número de individuos corresponde a 423 árboles por hectárea ( $\geq 10 \mathrm{~cm}$ dap), valor que se encuentra dentro del ámbito reportado en estudios realizados en otros sitios del país con características similares de altitud o precipitación que se encuentra entre 407 y 509 árboles/ ha (Cascante \& Estrada 2001, Di Stefano et al. 1996). En términos de área basal el valor obtenido para los bosques estudiados fue de $20,6 \mathrm{~m}^{2} / \mathrm{ha}$ con un diámetro promedio de $22,5 \mathrm{~cm}$, menor a la obtenida para el bosque muy húmedo premontano estudiado por Cascante y Estrada (2001) que reportan un área basal promedio de 36,4 m²/ ha; un diámetro promedio de $29,2 \mathrm{~cm}$ aproximadamente la mitad de la encontrada en el bosque muy húmedo premontano de la Reserva Biológica Alberto Brenes que reporta $41,7 \mathrm{~m}^{2} /$ ha (Wattenberg et al. 1996). Estas diferencias en los valores de área basal se sustentan en la distribución del número de individuos, la cual presenta estructura discetánea en forma de "J" invertida, que es un claro indicador del proceso de regeneración de las especies, en donde la acumulación del mayor número de individuos se da en los diámetros menores (10 a 30 $\mathrm{cm})$ y la presencia de pocos individuos de diámetros 
grandes. En consecuencia, la tendencia del área basal tiene un comportamiento similar a la encontrada para el número de individuos, ya que la acumulación se da en las categorías diamétricas inferiores y disminuye conforme aumenta el diámetro.

Para el piso montano bajo, los valores para el número de individuos en los bosques tanto de altura (380 árboles/ ha) como intervenidos (318 árboles/ha), es baja si se compara con los reportados en estudios realizados en otros bosques montanos del país, que se encuentran en un ámbito que va de los 455 a 555 árboles/ha (Blaser \& Camacho 1991, Jiménez et al. 1988, Nadkarni et al. 1995). En cuanto al área basal, sigue un comportamiento similar al presentado por la densidad, ya que tanto los bosques intervenidos $\left(15,7 \mathrm{~m}^{2} / \mathrm{ha}\right)$ como los bosques de altura $\left(27,2 \mathrm{~m}^{2} / \mathrm{ha}\right)$ presentaron valores muy por debajo de los reportados por otro autores, que van desde 36,7 a 64,7 $\mathrm{m}^{2}$ /ha (Blaser \& Camacho 1991, Orozco 1991 \& Kapelle 1996). Es importante destacar que el comportamiento de los robledales dispara los valores de área basal, ya que generalmente están compuestos de individuos con dimensiones muy grandes de diámetro y de altura.

La distribución diamétrica del número de árboles tanto para los bosques de altura como para los bosques intervenidos sigue una tendencia de "J invertida", y aunque la mayor cantidad de individuos se encuentre en las categorías diamétricas inferiores, hay una cantidad importante de individuos remanentes de diámetros grandes, al menos en los bosques de altura, lo que asegura, como se evidencia en la distribución del área basal, un incremento en el área basal que se mantiene en las clases diamétricas superiores, comportamiento asímil al encontrado en los bosques intervenidos en este mismo piso. Los picos de dominancia se dan en la clase de 20 a $30 \mathrm{~cm}$ influenciada directamente por la cantidad de individuos agrupados en esta clase diamétrica. Un incremento en el diámetro evidencia fuertes reducciones en el número de árboles y en este caso también en el área basal.

Se evaluaron además los potreros arbolados, siendo éstos una cobertura predominante en el entorno ecológico de la microcuenca, principalmente en los pisos altitudinales, premontano y montano bajo.

En el piso premontano, dicha cobertura reportó 121 árboles/ha, mientras que para el montano bajo 118 árboles/ha. Estos valores que difieren a los encontrados por Harvey y Haber (1999) en estudios realizados en fincas ganaderas en Monteverde, donde se encontró un intervalo de entre 5 a 80 árboles por hectárea

En lo que respecta al área basal, la dominancia reportada para los potreros arbolados corresponde $6,54 \mathrm{~m}^{2} /$ ha para el piso premontano y $6,99 \mathrm{~m}^{2} /$ ha para el piso montano bajo.

La distribución diamétrica del número de árboles, al igual que para los bosques, sigue una tendencia de jota invertida, sin embargo en la distribución del área basal se denota que dentro de estas coberturas se encontraron árboles remanentes de diámetros superiores, principalmente de $40-50 \mathrm{~cm}$ de diámetro.

\section{Estructura vertical}

La estructura vertical de los ecosistemas estudiados está representada por tres estratos, donde la tendencia de agrupar la mayor cantidad de individuos en el estrato medio se repite en todos los pisos altitudinales.

En el caso de los bosques intervenidos en el piso basal, poco más del $60 \%$ de los individuos se ubica en el piso medio (9-23 m), comportamiento similar al reportado por otros autores, donde el porcentaje de individuos en dicho estrato va de $44 \%$ a $45 \%$ (Suatunce et al. 2003, Morales 2010). Sin embargo, en estos bosques la representación en el piso inferior es alta, caso contrario a lo observado en los bosques intervenidos del piso basal, donde el piso inferior agrupa a menos del $10 \%$ de los individuos.

En el caso del piso premontano, los bosques intervenidos presentan las mayores alturas, con un estrato superior que alcanza hasta los $40 \mathrm{~m}$ de altura y un estrato medio que agrupa al $56 \%$ de los individuos, mientras que los potreros arbolados presentan un estrato más reducido que alcanza $25 \mathrm{~m}$ y donde el estrato medio agrupa al 45 $\%$ de los individuos.

Para el piso montano bajo, los bosques evaluados concentran la mayor cantidad de individuos en el estrato que va de $10-20 \mathrm{~m}$, con un estrato superior con pocos individuos, $22 \%$ para los bosques de altura y $8 \%$ para los bosques intervenidos. Por otro lado, los potreros en este piso altitudinal acumulan el $43 \%$ de sus individuos en el piso superior que va de los 14 a los $21 \mathrm{~m}$ de altura. Habitualmente, los robledales montanos consisten en rodales de más de $40 \mathrm{~m}$ de altura, con una estratificación vertical muy clara (Kappelle 2001), sin embargo, es importante tomar en cuenta que para este tipo de bosques, generalmente el dosel y el subdosel disminuyen de estatura con el incremento en la altitud (Kappelle et al. 1996).

En resumen y de acuerdo con el análisis de las clases de altura, los bosques estudiados en los diferentes pisos altitudinales presentan un piso medio denso, que puede llegar a limitar el establecimiento de individuos de otras especies con diferentes requerimientos lumínicos tanto en el piso inferior como en el superior.

\section{Conclusiones}

El paisaje de la microcuenca del Río La Balsa está compuesto por tres usos del suelo predominantes: bosques, pastos y cultivos agrícolas. 
Los ecosistemas más diversos correspondieron a los bosques intervenidos del piso basal y los menos diversos a los bosques de altura del piso montano bajo.

La estructura horizontal sugirió que los hábitats presentes en la microcuenca se encuentran en estado de recuperación.

La estructura vertical indicó que los hábitats estudiados presentaron un dosel medio denso.

\section{Recomendaciones}

Establecer un monitoreo con parcelas permanentes de muestreo para dar seguimiento a los cambios estructurales y de cobertura en los diferentes pisos altitudinales que componen la microcuenca del Río La Balsa.

Complementar con otros estudios, por ejemplo, análisis de intercepción de precipitaciones y cuantificación y evaluación de la percolación, erosión y sedimentación de suelos, ambos, para las coberturas predominantes en la microcuenca.

\section{Agradecimientos}

A la Compañía Nacional de Fuerza y Luz, específicamente al Departamento de Recursos Naturales por el apoyo en el desarrollo de esta investigación.

\section{Referencias}

Acosta, V.L. 2012. Análisis silvigénico de los bosques húmedos tropicales del Parque Nacional La Cangreja. Tesis de Lic. Cartago, Instituto Tecnológico de Costa Rica, Esc. de Ing. Forestal. $117 \mathrm{p}$.

Blaser, J., Camacho, M. 1991. Estructura, composición y aspectos silviculturales de un bosque de robles (Quercus spp.) del piso montano en Costa Rica. Turrialba, CR, CATIE Centro Agronómico Tropical de Investigación y Enseñanza. 67 p. (Colección Silvicultura y Manejo de Bosques Naturales $\mathrm{N}^{0} 1$ ).

Cascante, M., Estrada, A. 2001. Composición florística y estructura de un bosque húmedo premontano en el Valle Central de Costa Rica. (en línea). Revista de Biología Tropical 49(1):213-225. Consultado 14 mar. 2012. Disponible en http://www.scielo.sa.cr/scielo. php? pid=S003477442001000100020\&script $=$ sci_ abstract

Castro, S. 2008. Caracterización biofísica de la microcuenca del río Balsa. Alajuela, CR, CNFL Compañía Nacional de Fuerza y Luz . $101 \mathrm{p}$.

Chacón, C., Harvey, C. 2008. Contribuciones de las cercas vivas a la estructura y la conectividad de un paisaje fragmentado en Río Frío, Costa Rica. In Evaluación y conservación de biodiversidad en paisajes fragmentados de Mesoamérica. Eds. C Harvey; J Sáenz. Heredia, CR, INBio Instituto Nacional de Biodiversidad. p. 225-250.
Di Stéfano, J.F., Nielsen, V., Hoomans, J., Fournier, L.A. 1996. Regeneración de la vegetación arbórea en una pequeña reserva forestal urbana del nivel premontano húmedo, Costa Rica. (en línea). Revista de Biología Tropical 44(2):575-580. Consultado 14 mar. 2012. Disponible en http://www.biologiatropical.ucr.ac.cr/attachments/ volumes/vol442A/25_Di_Stefano_Vegetacion_arborea. pdf.

Di Rienzo, J.A., Casanoves, F., Balzarini, M.G., González, L., Tablada, M., Robledo, C.W. InfoStat. 2011. (en línea). AR, Grupo InfoStat, Universidad Nacional de Córdoba.

Elkie, P.C., Rempel, R.S., Carr, A. 1999. Patch analyst user's manual: A tool for quantifying landscape structure. Ontario, CA, Queen's printer for Ontario. 23 p. (Northwest Science \& Technology Technical Manual TM-002).

FAO (Organización de las Naciones Unidas para la Agricultura y la Alimentación, IT). 2003. Situación de los bosques del mundo. Roma, IT, FAO. p.1-22.

Gallego, B. 2002. Estructura y composición de un paisaje fragmentado y su relación con especies arbóreas indicadoras en una zona de bosque muy húmedo tropical, Costa Rica. Tesis M.Sc. Turrialba, CR, CATIE Centro Agronómico Tropical de Investigación y Enseñanza. 116 p.

Hammer O., Harper, D., Ryan, P. 2001. Paleontological statistics software package for education and data analysis. (en línea). Paleontología Electrónica 4(1):1-9. Consultado 19 mar. 2011. Disponible en http://palaeoelectronica. org/2001_1/past/past.pdf

Harvey, C., Haber, W. 1999. Remnant trees and the conservation of biodiversity in Costa Rica. Ecological Applications 10:155-173.

Harvey, C.A., Villanueva, C.; Ibrahim, M., Gómez, R., López, M., Kunth, S., Sinclair, F. 2004. Productores, árboles y producción ganadera en paisajes de América Central: Implicaciones para la conservación de la biodiversidad. In Harvey, C y Sáenz, J. (Eds.). Evaluación y conservación de biodiversidad en paisajes fragmentados de Mesoamérica. Heredia, CR, INBio Instituto Nacional de Biodiversidad. p. 197-224.

Harvey, C., Guindon, C., Haber, W., Hamilton, D; Murray, K. 2005. Importancia de los fragmentos de bosque, los árboles dispersos y las cortinas rompevientos para la biodiversidad local y regional: el caso de Monteverde, Costa Rica. In Harvey, C y Sáenz, J. (Eds.). Evaluación y conservación de biodiversidad en paisajes fragmentados de Mesoamérica. Heredia, CR, INBio Instituto Nacional de Biodiversidad. p. 289-326.

Harvey, C., Sáenz, J. 2008. Eds. Evaluación y conservación de biodiversidad en paisajes fragmentados de Mesoamérica. Heredia, CR, INBio Instituto Nacional de Biodiversidad. $624 \mathrm{p}$.

Holdridge, L.R. 1987. Ecología basada en zonas de vida. San José, CR, IICA Instituto Interamericano de Cooperación ara la Agricultura. $216 \mathrm{p}$.

Huber, A., Echeverria, C., Taberlet, F. 2007. Estudio comparativo de los componentes del balance hídrico en un bosque nativo y una predera en el sur de Chile. Bosque 28 (3): 271-280. Consultado 12 mar. 2012. Disponible en http:// mingaonline.uach.cl/pdf/bosque/v28n3/art13.pdf. 
Iroumé, A., Huber, A. 2000. Intercepción de las lluvias por la cubierta de bosques y efecto en los caudales de crecida en una cuenca experimental en Malalcahuello, IX Región, Chile. Bosque 21(1): 45-56. Consultado 12 mar. 2012. Disponible en http://cybertesis.uach.cl/tesis/uach/2011/ fifb826r/doc/fifb826r.pdf.

ITCR (Instituto Tecnológico de Costa Rica). 2008. Atlas digital de Costa Rica 2008. (CD-ROM). Cartago, Instituto Tecnológico de Costa Rica, Esc. de Ing. Forestal. 1 CD-ROM.

Jang, J.C., Nishigami, Y., Yanagisawa, Y. 1996. Assessment of global forest change between 1986 and 1993 using satellite-derived terrestrial net primarily productivity. Environmental Conservation 23 (4):315-321.

Jiménez, W., Chaverri A., Miranda, R., Rojas, I. 1988. Aproximaciones silviculturales al manejo de un robledal (Quercus spp.) en San Gerardo de Dota, Costa Rica. Turrialba 38(3):208-214.

Kaimowitz, D. 2001. Will livestock intensification help save Latin America tropical forest? In Agricultural technologies and tropical deforestation. Eds. A Angelsen; D Kaimowitz, Wallingford, UK, CABI. 1-20 p.

Kappelle, M. 2001. Bosques nublados del neotrópico. Eds. M. Kappelle; A. Brown. Heredia, CR, INBio Instituto Nacional de Biodiversidad. 704 p.

Kapelle, M. 1996. Los Bosques de Roble (Quercus) de la Cordillera de Talamanca, Costa Rica: Biodiversidad, ecología, conservación y desarrollo. Heredia, CR, Universidad de Amsterdam-INBio. p. 1-112.

Lamprecht, H. 1990. Silvicultura en los trópicos: Los ecosistemas forestales en los bosques tropicales y sus especies arbóreas-posibilidades y métodos para un aprovechamiento sostenido. Trad. A. Carrillo. Eschborn, DE. GTZ. p. 60-62.

Lieberman, D., Hartshorn, G.S., Lieberman, M., Peralta, R.. 1990. Forest dynamics of La Selva Biological Station, 1969-1985. In Four Neotropical Rainforest. Ed. A. Gentry. New Haven, Connecticut, US, Yale University. p. 509521.

Medianero, E., Valderrama, A., Barrios, H. 2003. Diversidad de insectos minadores de hojas y formadoras de agallas en el dosel y sotobosque del bosque tropical. (en línea). Consultado 30 abr. 2012. Disponible en http://redalyc. uaemex.mx/src/inicio/ArtPdfRed.jsp?iCve=57508912

Melo, O., Vargas, R. 2003. Evaluación ecológica y silvicultural de ecosistemas boscosos. Ibagué, CO, Universidad de Tolima. $183 \mathrm{p}$.

Morales, M. 2010. Composición florística, estructura, muestreo diagnóstico y estado de conservación de una cronosecuencia de bosques tropicales del Corredor Biológico Osa, Costa Rica. Tesis de Lic. Cartago, CR, Instituto Tecnológico de Costa Rica. Esc., de Ing. Forestal. p15.

Morera, C., Pintó, J., Romero, M. 2007. Paisaje, procesos de fragmentación y redes ecológicas: aproximación conceptual. In Corredores Biológicos: acercamiento conceptual y experiencias en América. Eds. O. Chassot; C. Morera. San José, CR, Centro Científico Tropical y Universidad Nacional. $128 \mathrm{p}$.
Nadkarni, N.M., Matelson, T.J., Haber, W.A. 1995. Structural characteristics and floristic composition of a neotropical cloud forest, Monteverde, CR. Journal of Tropical Ecology 11:481-495.

Orozco, L. 1991. Estudio ecológico y de estructura horizontal de seis comunidades boscosas en la Cordillera de Talamanca, Costa Rica. Informe Técnico 176. Col. Silvic. Man. Bosq. Nat. 2. Turrialba, CR, CATIE Centro Agronómico Tropical de Investigación y Enseñanza. 35 p.

PROIGE (Programa de Investigación en Geografía, CR). 2007. Base de datos geográfica y elaboración de cartografía de la microcuenca del río La Balsa. San José, Universidad de Costa Rica, PROIGE. 47 p.

Quesada, R. 2003. Dinámica del crecimiento diamétrico del bosque tropical intervenido de bajura. Cartago, Instituto Tecnológico de Costa Rica, Esc. de Ing. Forestal. (en línea). Consultado 2 mayo 2012. Disponible en http:// www.una.ac.cr/inis/docs/silvic/Quesa2.pdf

Ramírez, P. 2009. Balance hídrico de suelos de la cuenca del río Balsa. San José, CR, CNFL Compañía Nacional de Fuerza y Luz. $101 \mathrm{p}$.

Sánchez, G. 2001. Deforestation in Costa Rica: A quantitative analysis using remote sensing imagery. Biotrópica 33(3):378-384

Saunders, D.A., Hobbs, R..J., Margules, C.R.. 1991. Biological consequences of ecosystem fragmentation: A review. Conservation Biology 5:18-32.

Skoles, D., Tucker, C. 1993. Tropical deforestation and habitat fragmentation in the Amazon: Satellite data from 1978 to 1988. Science 260:1905-1908.

Suatunce, P., Somarriba, E., Harvey, C., Finegan, B. 2003. Composición florística y estructura de bosques y cacaotales en los territorios indígenas de Talamanca, Costa Rica. (en línea). Agroforestería en las Américas 10(37-38):31-35. Consultado 11 mar. 2012. Disponible en http://worldcocoa.com/scientificresearch/researchlibrary/documents/Suatunce2003.pdf

Thomsen, K. 1997. Potential of non-timber forest products in tropical rain forest in Costa Rica. Ph.D. Tesis. Copenhagen, DK, University of Copenhagen, Faculty of Natural Sciences. P.20-42.

Vílchez, B., Chazdon R., Milla, V. 2008. Dinámica de la regeneración en cuatro bosques secundarios tropicales de la región Huetar Norte, Costa Rica: Su valor para la conservación o uso comercial. Recur. Nat. Ambient 55:118-128.

Wattenberg, I., Breckle, S., Ortiz, R.. 1996. La diversidad de especies arbóreas y la estructura de un bosque muy húmedo premontano en la Reserva Biológica Alberto Manuel Brenes. Rev. Pensamiento Actual 2:11-19. 